\title{
Autorinnen und Autoren / List of Contributors
}

Prof. Dr. John Duffy

Harvard University

Department of the Classics

204 Boylston Hall

Cambridge, MA 02138, U.S.A.

duffy2@fas.harvard.edu

Prof. Dr. Stephanos Efthymiadis

University of Ioannina

Department of Philology

GR - 45110 Ioannina

sefthym@,cc.uoi.gr

Dr. J. Featherstone

College de France

Centre d'Etudes byzantines

52 rue du Cardinal Lemoine

F - 75005 Paris

m.featherstone@,college-de-france.fr

Dr. Fritz Felgentreu

Freie Universität Berlin

Seminar für Klassische Philologie

Ehrenbergstraße 35

D - 14195 Berlin-Dahlem

ffelge@,zedat.fu-berlin.de

Dr. Niels Gaul

Lincoln College

Oxford OX1 3DR, U.K.

niels.gaul@classics.ox.ac.uk

Dr. Michael Grünbart

Universität Wien

Fachbereichsbibliothek Byzantinistik

und Neogräzistik

Postgasse 7

A - 1010 Wien

michael.gruenbart@univie.ac.at
Lars Hoffmann M.A.

Johannes Gutenberg-Universität Mainz

FB-07 Geschichts- und

Kulturwissenschaften

Historisches Seminar Abt. 5

Byzantinistik

D - 55099 Mainz

lhoffman@uni-mainz.de

Dr. Grammatiki Karla

Neapoleos 116

GR - 15341 Agia Paraskevi

grammatikikarla@hotmail.com

Prof. Dr. Sofia Kotzabassi

Aristotle University of Thessaloniki

Department of Medieval and Modern

Greek Philology

GR - 54006 Thessaloniki

kotzabas@lit.auth.gr

Prof. Dr. Stavros Kourouses

Perikleous 96

GR - 15231 Kato Chalandrion /Athen

Dr. Gernot Krapinger

Karl-Franzens-Universität Graz

Institut für Klassische Philologie

Universitätsplatz 3/II

A - $8010 \mathrm{Graz}$

gernot.krapinger@uni-graz.at

Prof. Dr. Tilman Krischer

Straße zum Löwen 24

D - 14109 Berlin

Prof. Dr. Ralph-Johannes Lilie

Berlin-Brandenburgische Akademie

Prosopographie der

mittelbyzantinischen Zeit

Jägerstraße 22/23

D - 10117 Berlin

lilie@bbaw.de 
Prof. Dr. Antony Littlewood

The University of Western Ontario

Department of Classical Studies, Talbot

College

London, Ontario N6A 3K7, Canada

splinter@uwo.ca

Prof. Dr. Marina Loukaki

University of Crete

Department of Philology

GR - 74100 Rethymno

loukaki@phl.uoc.gr

Dr. Przemysław Marciniak

Wissenschaftskolleg zu Berlin

Institute for Advanced Study

Wallotstraße 19

D - 14193 Berlin

przemyslaw.marciniak@,WiKo-

Berlin.DE

PD Dr. Karin Metzler

Theologische Fakultät der Humboldt-

Universität

Lehrstuhl für Ältere Kirchengeschichte

Hausvogteiplatz 8

D - 10117 Berlin

karin.metzler@,rz.hu-berlin.de

PD Dr. Dietmar Najock

Freie Universität Berlin

Seminar für Klassische Philologie

Ehrenbergstraße 35

D - 14195 Berlin

Prof. Dr. Eustratios Papaioannou

Brown University

Department of Classics

Macfarlane House, 48 College Street

Providence, RI 02912, U.S.A.

Eustratios_Papaioannou@brown.edu
PD Dr. Thomas Pratsch

Berlin-Brandenburgische Akademie

Prosopographie der

mittelbyzantinischen Zeit

Jägerstraße 22/23

D - 10117 Berlin

thomas_pratsch@,web.de

Dr. Andreas Rhoby

Österreichische Akademie der

Wissenschaften

Institut für Byzanzforschung

Postgasse 7/1/3

A - 1010 Wien

andreas.rhoby@oeaw.ac.at

Dr. Andrew F. Stone

The University of Western Australia

Classics and Ancient History

Crawley W.A. 6009, Australia

afstone@cyllene.uwa.edu.au

Dr. Ida Toth

The Classics Centre

George Street

Oxford OX1 2R1, UK

ida.toth@,wolfson.oxford.ac.uk

Prof. Dr. Erich Trapp

Friedrich-Wilhelms-Universität Bonn

Philologisches Seminar

Am Hof 1e

D - 53115 Bonn

erich.trapp@uni-bonn.de

Dr. Ruth Webb

University of London/Birkbeck College

School of History, Classics and

Archaeology

Malet Street

London WC1E 7HX, U.K.

r.webb@history.bbk.ac.uk 

